\title{
Singularities at Infinity and their Vanishing Cycles, II. Monodromy
}

By

\author{
Dirk SiersmA* and Mihai TibĂR**
}

\begin{abstract}
Let $f: \mathbb{C}^{n} \rightarrow \mathbb{C}$ be any polynomial function. By using global polar methods, we introduce models for the fibers of $f$ and we study the monodromy at atypical values of $f$, including the value infinity. We construct a geometric monodromy with controlled behavior and define global relative monodromy with respect to a general linear form. We prove localization results for the relative monodromy and derive a zeta-function formula for the monodromy around an atypical value. We compute the relative zeta function in several cases and emphasize the differences to the "classical" local situation.
\end{abstract}

\section{$\S 1$. Introduction}

We study a polynomial function $f: \mathbb{C}^{n} \rightarrow \mathbb{C}$ aiming to describe the variation of topology in the fibration induced by $f$, at atypical fibers. The topology of polynomial functions became a challenging topic after the paper of Broughton $[\mathrm{Br}]$ since applying local methods encounters obstructions from the non properness of $f$ and from the asymptotic nongenericity. In two variables, the interest increases by the reduction of the Jacobian Conjecture to the following statement: if $f$ has singularities at infinity then the singular locus $\operatorname{Sing}(f, g)$ is not

Communicated by K. Saito, February 27, 1999. Revised December 24, 1999.

2000 Mathematics Subject Classification(s): 32S40, 32S50, 32S15.

Key words: topology of polynomial functions, singularities at infinity, relative monodromy.

* Mathematisch Instituut, Universiteit Utrecht, PO Box 80010, 3508 TA Utrecht, The Netherlands.

e-mail: siersma@math.uu.nl

** Mathématiques, UMR 8524 CNRS, Université de Lille 1, 59655 Villeneuve d'Ascq, France.

e-mail: tibar@agat.univ-lille1.fr 
empty, for any polynomial function $g$ [LW], [ST]. The problem raised by such a statement is how to control the so called "singularities at infinity". As we show in this paper, they represent a new type of singularities, their behavior being different from the one of local singularities. In several variables, the term "singularities at infinity" is well defined only under specific conditions and possibilities of study are limited because these singularities can be non isolated. In two variables, only isolated singularities at infinity can occur and one mav define them in several equivalent ways.

In case $f$ has isolated singularities at infinity (in the sense that the proper extension $p: \mathbb{X}^{\prime} \rightarrow \mathbb{C}$, defined in $\S 2$, has isolated singularities with respect to a Whitney stratification of the space $\mathbb{X}^{\prime}$ ) we have introduced in $[\mathrm{ST}]$ the "vanishing cycles at infinity" and proved that they generate the homology of the generic fiber together with the cycles vanishing at critical points in the affine. It appears that there is an interaction between the two types of cycles and that this interaction is of a new type, different from the one between cycles vanishing in the affine.

Starting from our previous results [ST], [Ti-3], [Ti-4]. we develop in this paper a method for studying the monodromy of $f$ via the relative monodromy. The motivation for looking to relative monodromy is that, by slicing, we get a polynomial in less coordinates and with less singularities (at least those at infinity), see the proof of Proposition 4.8.

There are recent papers which contain results on some invariants of the monodromy, usually under certain restrictions on $f$. Different points of view are adopted: Newton polyhedra [LS], Hodge theory of families of algebraic hypersurfaces [GN], Fourier transform of $\mathcal{D}$-modules [Sa], resolution of singularities $[\mathrm{MW}],[\mathrm{ACD}],[\mathrm{GLM}]$, relative monodromy in case of two variables [Ha].

Our approach is via the construction of geometric monodromies along loops in $\mathbb{C}$ and has been announced in [ST-2]. We first recall from [ $\mathrm{Ti}-4]$ the construction of a model of a fiber of $f$, which uses a tomographic method, the slicing with generic hyperplanes. Repeating this procedure in lower dimensions, one arrives at a generic skeleton. This idea was first used by the second author for proving that a certain asymptotic equisingularity condition is controlled by global polar invariants, in case of a family of affine hypersurfaces [Ti-4].

Then we define a global geometric monodromy, i.e. a representation $\rho$ : $\pi_{1}\left(\mathbb{C} \backslash \Lambda_{f}\right) \rightarrow \operatorname{Diff}(F)$, where $\Lambda_{f}$ is the set of atypical values, $F$ is the general fiber of $f$ and $\operatorname{Diff}(F)$ is the group of $\mathrm{C}^{\infty}$-diffeomorphisms of $F$. In case of a monodromy at an atypical value (including the value $\infty \in \mathbb{P}^{1}$ ) we define a certain vector field, controlled at infinity and tangent to the polar curve 
$\Gamma(l, f):=\operatorname{closure}\{\operatorname{Sing}(l, f) \backslash \operatorname{Sing} f\}$ of $f$ with respect to a general linear form $l$. The construction yields a refined filtered structure of the affine fiber $F$, reflecting the dynamic behavior of each piece in the decomposition of $F$, when turning around the atypical value.

This also represents a globalization of the local carrousel construction of Lê D.T. [Lê-1], [Lê-2]. We prove localization results for the relative monodromy which lead to a zeta function formula for any $f$. We emphasize on the new features that occur in the neighbourhood of infinity: there are certain loops around infinity which enter in the description of the relative monodromy. These loops are in particular the cause of the error in the main result of Hà H.V.'s paper [Ha, Theorem 3.4]. Namely, in case $n=2$, at a singular point at infinity with $\lambda=1$ (called "Morse singularity at infinity" in loc cit) the local monodromy can effectively be either +id, or -id, see Examples 6.1 and 6.2. This does not happen in the local case: the monodromy of a local Morse curve singularity is allways + id. Examples of computations of the relative monodromy are included at the end.

\section{Acknowledgements}

Part of this research was done as a RiP program at Oberwolfach, which was supported by the Volkswagen-Stiftung. The authors benefited from the excellent working conditions offered by the Mathematisches Forschungsinstitut Oberwolfach. They thank the referee for remarks that contributed to improve the exposition.

\section{$\S 2 . \quad$ Models of Fibers}

Let $f: \mathbb{C}^{n} \rightarrow \mathbb{C}$ be a polynomial function of degree $d$. A value $t_{0} \in \mathbb{C}$ is called typical if $f$ is a trivial $\mathrm{C}^{\infty}$-fibration at $t_{0}$. It is known that the set of atypical values, denoted $\Lambda_{f}$, is finite (because of algebraicity) cf. [T], [Va], [Vej. It includes the critical values of $f$ but also other values, the fibers over which have singular asymptotic behavior. Under certain conditions, one can localize the variation of topology at atypical values, thus obtaining "singular points at infinity", cf. [Ti-3]. We need for that a support at infinity, to contain all the "ends" of fibers of $f$.

We consider the closure of the graph of $f$ in $\mathbb{P}^{n} \times \mathbb{P}^{1}$, namely the space $\mathbb{X}=\left\{\left[x_{0}: x_{1}: \cdots x_{n}\right] \in \mathbb{P}^{n},[s: t] \in \mathbb{P}^{1} \mid s \tilde{f}-t x_{0}^{d}=0\right\} \subset \mathbb{P}^{n} \times \mathbb{P}^{1}$, where $\tilde{f}$ is the homogenized of the polynomial $f$. Here and in the following $x_{0}$ denotes the variable at infinity, defining the embedding $\mathbb{C}^{n} \subset \mathbb{P}^{n}$. Denote by $p: \mathbb{X} \rightarrow \mathbb{P}^{1}$ the second projection and by $\mathbb{X}^{\infty}:=\mathbb{X} \cap\left\{x_{0}=0\right\}$ the hyperplane at infinity of 
$\mathbb{X}$. Since the graph of $f$ is diffeomorphic to the domain, we have an embedding $i: \mathbb{C}^{n} \simeq \operatorname{Graph}(f) \hookrightarrow \mathbb{X}$. The map $f$ is the composition $\mathbb{C}^{n} \stackrel{\iota}{\hookrightarrow} \mathbb{X} \stackrel{p}{\rightarrow} \mathbb{C}$. So the study of $f$ is equivalent to the study of the restriction of $p$ to the graph of $f$.

There is a finite set $\Lambda \subset \mathbb{P}^{1}, \Lambda \supset \Lambda_{f}$, minimal with the property that the restriction $p_{\mid}: \mathbb{X} \backslash p^{-1}(\Lambda) \rightarrow \mathbb{P}^{1} \backslash \Lambda$, resp. $p_{1}: \mathbb{X} \backslash\left(p^{-1}(\Lambda) \cup \mathbb{X}^{\infty}\right) \rightarrow \mathbb{P}^{1} \backslash \Lambda$, is a $\mathrm{C}^{0}$, resp. $\mathrm{C}^{\infty}$, locally trivial fibration $[\mathrm{T}],[\mathrm{Ve}]$. We take by definition $[0: 1]=$ $\infty \in \Lambda$. We consider the polar locus $\Gamma(l, f):=\operatorname{closure}\{\operatorname{Sing}(l, f) \backslash \operatorname{Sing} f\} \subset \mathbb{C}^{n}$ of $f$ with respect to a linear form $l: \mathbb{C}^{n} \rightarrow \mathbb{C}$ and denote by $\Delta:=(l, f)(\Gamma(l, f))$ the polar image in $\mathbb{C}^{2}$. Denote by $l_{H}$ the linear form associated to a projective hyperplane $H \in \check{\mathbb{P}}^{n-1}$. We first prove the following key result.

Lemma 2.1. $\quad$ There is a Zariski-open set $\Omega \subset \check{\mathbb{P}}^{n-1}$ such that, for any $H \in \Omega$ :

(a) the polar locus $\Gamma\left(l_{H}, f\right)$ is a reduced curve or it is empty.

(b) the map $\left(l_{H}, f\right): \mathbb{C}^{n} \rightarrow \mathbb{C} \times \mathbb{C}$ is a $\mathrm{C}^{\infty}$-trivial fibration over $\left(\mathbb{C} \times\left(\mathbb{C} \backslash \Lambda_{H}\right)\right) \backslash$ $\Delta$, where $\Lambda_{H}$ is some finite set in $\mathbb{C}$.

Proof. (a) is a slightly improved version of the Polar Curve Theorem, see [Ti-3, Lemma 2.4]. That the polar curve is reduced follows from standard Bertini type arguments. We prove (b) in the following. Take a finite complex stratification $\mathcal{W}:=\left\{\mathcal{W}_{\imath}\right\}_{\imath \in I}$ of $\mathbb{X}^{\prime}:=\mathbb{X} \cap\left(\mathbb{P}^{n} \times \mathbb{C}\right)$ satisfying Whitney conditions and such that $\mathbb{C}^{n} \subset \mathbb{X}^{\prime}$ is a stratum. (The embedding of $\mathbb{C}$ into $\mathbb{P}^{1}$ is given by making $s=1$ and the embedding of $\mathbb{C}^{n}$ into $\mathbb{X}^{\prime}$ comes from the isomorphism $\mathbb{C}^{n} \simeq \operatorname{Graph}(f)$ and the embedding of the graph of $f$ into $\mathbb{X}$, as defined above.) There is a canonical Whitney stratification with this property, cf. [Te].

We take two copies of $\mathbb{C}$, denoted $\mathbb{C}_{r}$ and $\mathbb{C}_{t}$, of variables $r$, respectively $t$. Let $\mathbb{H}:=\left\{\left(r,\left[x_{0}: x\right], t\right) \in \mathbb{C}_{r} \times \mathbb{P}^{n} \times \mathbb{C}_{t} \mid l_{H}-r x_{0}=0\right\}$ (notice that $t$ is free). Consider $\mathbb{X}^{\prime}$ as subset of $\mathbb{P}^{n} \times \mathbb{C}_{t}$. Note that $\mathbb{H}$ is nonsingular, whereas $\mathbb{X}^{\prime}$ can have singularities on $\mathbb{X}^{\infty}$. Define the space:

$$
\mathbf{Y}:=\left(\mathbb{C}_{r} \times \mathbb{X}^{\prime}\right) \cap \mathbb{H} \subset \mathbb{C}_{r} \times \mathbb{P}^{n} \times \mathbb{C}_{t}
$$

and consider the projection $(r, t): \mathbf{Y} \rightarrow \mathbb{C}_{r} \times \mathbb{C}_{t}$. This is a proper extension of the map $(l, f)$. It follows that $Y \backslash Y^{\infty}$ is non-singular (since it can be identified with $\left.\mathbb{C}^{n}\right)$, where $\mathbf{Y}^{\infty}:=\mathbf{Y} \cap\left(\mathbb{C}_{r} \times\left\{x_{0}=0\right\} \times \mathbb{C}_{t}\right)$, and that the critical locus of the restriction $(r, t)_{\mid \mathbf{Y} \backslash \mathbf{Y}^{\infty}}$ is $\operatorname{Sing}(l, f)$. It remains to understand the situation at infinity. 
We first return to the space $\mathbb{X}^{\prime}$ and the map $p: \mathbb{X}^{\prime} \rightarrow \mathbb{C}$. Denote by Sing ${ }_{\mathcal{W}} p:=\cup_{\mathcal{W}_{\imath} \in \mathcal{W}} \operatorname{Sing} p_{\mid \mathcal{W}_{\imath}}$ the singular locus of $p$ with respect to the stratification $\mathcal{W}$. It is a closed analytic subset of $\mathbb{X}^{\prime}$.

For a fixed stratum $\mathcal{W}_{i} \subset \mathbb{X}^{\prime} \cap \mathbb{X}^{\infty} \subset \mathbb{P}^{n-1} \times \mathbb{C}_{t}$, we consider the projectivized relative conormal $\mathbb{P} T_{p \mid \mathcal{W}_{2}}^{*} \subset \mathbb{P}^{n-1} \times \mathbb{C}_{t} \times \check{\mathbb{P}}^{n-1}$. For the definition of the relative conormal, we refer to $[\mathrm{Te}],[\mathrm{HMS}]$. Let us just emphasize that there is a unique hyperplane within $\mathbb{P}^{n-1}$ which is tangent to a given non-singular point of some fiber of $p_{\mid \mathcal{W}_{\imath}}$ and we identify it with an element of $\check{\mathbb{P}}^{n-1}$.

We remark that, if $p$ is not constant on $\mathcal{W}_{\imath}$, then $\operatorname{dim} \mathbb{P} T_{p \mid \mathcal{W}_{\imath}}^{*}=n-1$. By using a Bertini type argument for the projection $\mathbb{P} T_{p \mid \mathcal{W}_{\imath}}^{*} \rightarrow \check{\mathbb{P}}^{n-1}$, it follows that there exists a Zariski open set $\Omega \subset \check{\mathbb{P}}^{n-1}$ such that, for any $H \in \Omega$, the map $p_{\mid \mathcal{W}_{\imath}}$ restricted to $\mathcal{W}_{\imath} \cap\left(H \times \mathbb{C}_{t}\right)$ is a submersion at all points except of the singular locus Sing $\mathcal{W}^{p}$ and except of at most a finite set $A_{H}$, for any $i \in I$. We may and shall assume, without loss of generality (after eventually intersecting with a Zariski open set), that the set $\Omega$ also verifies the point (a) of our lemma.

Now let us construct a Whitney stratification of $\mathbf{Y}$. We take $\mathbf{Y} \backslash \mathbf{Y}^{\infty} \simeq \mathbb{C}^{n}$ as a stratum and start to stratify $\mathbf{Y}^{\infty}$. Consider the product stratification $\mathbb{C}_{r} \times \mathcal{W}^{\prime}$ of $\mathbb{C}_{r} \times \mathbb{X}^{\prime}$, where $\mathcal{W}^{\prime} \subset \mathbb{X}^{\prime} \cap \mathbb{X}^{\infty}$. This is a Whitney stratification too. The intersection of $\mathbb{C}_{r} \times \mathcal{W}_{\imath}$ with $\mathbb{H}$ is transverse within $\mathbb{C}_{r} \times \mathbb{P}^{n} \times \mathbb{C}_{t}$ if and only if the intersection $\left(\mathbb{C}_{r} \times \mathcal{W}_{\imath}\right) \cap\left(\mathbb{C}_{r} \times H \times \mathbb{C}_{t}\right)$ is transverse within $\mathbb{C}_{r} \times \mathbb{P}^{n-1} \times \mathbb{C}_{t}$. This latter is indeed transverse for any $i$, at all points except of the set $\mathbb{C}_{r} \times A_{H}$, if $H \in \Omega$. Leaving aside the exceptional set $\mathbb{C}_{r} \times A_{H}$, the transverse slices become Whitney strata of $\mathbf{Y}$ at infinity, since Whitney property is preserved by transverse cutting.

Next we have to see whether the fibres of the map $(r, t)$ are transverse to the above defined Whitney strata of $\mathbf{Y}$. Namely, the map $\left(\mathbb{C}_{r} \times \mathcal{W}_{i}\right) \cap$ $\left(\mathbb{C}_{r} \times H \times \mathbb{C}_{t}\right) \stackrel{(r, t)}{\rightarrow} \mathbb{C}_{r} \times \mathbb{C}_{t}$ is a submersion whenever the second projection $\mathcal{W}_{\imath} \cap\left(H \times \mathbb{C}_{t}\right) \stackrel{t}{\rightarrow} \mathbb{C}_{t}$ is a submersion. This situation is treated above, since the map $t$ coincides with the map $p$. The result is that, if $H \dot{\in} \Omega$, then the fibres of $(r, t)$ are transverse to the Whitney stratification of $\mathbf{Y}$ except at the points of the following set:

$$
\Sigma_{H}:=\Gamma\left(l_{H}, f\right) \cup\left(\mathbb{C}_{r} \times\left(\operatorname{Sing}_{\mathcal{W}} p \cup A_{H}\right)\right)
$$

Note that $t\left(\mathbb{C}_{r} \times \operatorname{Sing}{ }_{\mathcal{W}} p\right)$ is a finite set. Moreover, for $H \in \Omega, \Gamma\left(l_{H}, f\right) \cup\left(\mathbb{C}_{r} \times\right.$ $\left.A_{H}\right)$ is a curve (or empty). Denote by $\Delta_{H}(r, t) \subset \mathbb{C}_{r} \times \mathbb{C}_{t}$ the image of $\Sigma_{H}$ by $(r, t)$. We have thus proved that the map:

$$
(r, t)_{\mid}: \mathbf{Y} \backslash(r, t)^{-1}\left(\Delta_{H}(r, t)\right) \rightarrow\left(\mathbb{C}_{r} \times \mathbb{C}_{t}\right) \backslash \Delta_{H}(r, t)
$$


is a locally trivial, stratified fibration. Restricting it to $\mathbb{C}^{n}$, yields the desired statement (b). We may therefore define $\Lambda_{H}$ as $t\left(\mathbb{C}_{r} \times\left(\operatorname{Sing}{ }_{w} p \cup A_{H}\right)\right)$. Note that the set of critical values $\Lambda_{f}$ and the set of atypical values $\Lambda$ are contained in $\Lambda_{H}$.

When focusing to a certain (atypical) value $a \in \mathbb{C}$, we need to look at the special set of points $\Gamma\left(l_{H}, f\right) \cap F_{a}$, where $F_{a}$ denotes the fibre $f^{-1}(a)$. If such a point is a smooth point of $F_{a}$ then $f$ is locally trivial at this point but the map $\left(l_{H}, f\right)$ is not. We need to change $H$ in order to have transverse intersection of $\Gamma$ with $F_{a}$ at the point. This is possible as shown in the next lemma, which is a supplement of Lemma 2.1. If so, then we have a better situation along $F_{a}$ since transverse intersections provide local stratified product structures with respect to $\left(l_{H}, f\right)$, see Remark $4.6(\mathrm{~b})$.

Lemma 2.2. Let $a \in \mathbb{C}$. There is a Zariski-open set $\Omega_{a} \subset \Omega \subset \check{\mathbb{P}}^{n-1}$ such that, for any $H \in \Omega_{a}$, we have:

(c) as a hyperplane of the hyperplane at infinity $\mathbb{P}^{n-1}, H$ is transverse to all the strata at infinity of the canonical Whitney stratification of the projective hypersurface $p^{-1}(a) \subset \mathbb{P}^{n}$.

(d) the polar curve $\Gamma\left(l_{H}, f\right)$ has no component included into $f^{-1}(a)$.

(e) the restriction $l_{H \mid f^{-1}(a)}: f^{-1}(a) \rightarrow \mathbb{C}$ has only stratified Morse critical points outside the one point strata of the stratification considered at (c).

Proof. Condition (c) is an open condition and implies condition (d). Condition (e) is known to be locally open, hence open.

Convention 2.3. When $a=\infty$ (that is $\left.a=[0: 1] \in \mathbb{P}^{1}\right)$, we define $\Omega_{\infty}$ to be just $\Omega$ (the Zariski-open set from Lemma 2.1).

The following is an immediate consequence of the definition:

Corollary 2.4. Let $H \in \Omega$. If $F_{a}$ has an isolated singularity at $c \in F_{a}$ then $c \in \Gamma\left(l_{H}, f\right)$. More generally, if the restriction of $l_{H}$ on $F_{a}$ has an isolated stratified singularity at $c \in F_{a}$, then $c \in \Gamma\left(l_{H}, f\right)$.

We recall from $[\mathrm{Ti}-4,3.6]$ the description of the "tomographic" model of $F_{a}:=f^{-1}(a)$. According to Lemma 2.2, for $H \in \Omega_{a}$ the linear function $l_{H}: F_{a} \rightarrow \mathbb{C}$ is a fibration without singularities at infinity. The only critical 
points of $l_{H}: F_{a} \rightarrow \mathbb{C}$ are the intersection points $\Gamma\left(l_{H}, f\right) \cap F_{a}=\left\{c_{1}, \ldots, c_{k}\right\}$. By Lefschetz-Morse-Smale theory, the hypersurface $F_{a}$ is built from the slice $F_{a} \cap H$ by attaching a number of cells of dimension $n-1$ only. In case $F_{a}$ is singular, this result is still true by using the theory of functions with isolated singularities on stratified spaces, see [Lê-3]. To each point there corresponds an attaching of a number of $(n-1)$-cells equal to the $(n-2)^{\text {th }}$ Betti number of the local Milnor fiber $M_{H, c_{\imath}}$ of the germ $l_{H}:\left(F_{a}, c_{\imath}\right) \rightarrow \mathbb{C}$.

\subsection{Fibres with isolated singularities: the generic skeleton}

Let us be more explicit in case when $F_{a}$ is non-singular or it has only isolated singularities. By Milnor's Theorem, the Milnor fiber $M_{H, c_{\imath}}$ is homotopically a bouquet of spheres and by Lê's Theorem [Lê-3], their number is equal to the intersection multiplicity $\operatorname{int}_{c_{\imath}}\left(\Gamma\left(l_{H}, f\right),\left\{l_{H}=l_{H}\left(c_{\imath}\right)\right\}\right)$, which in turn is equal to the multiplicity mult $_{c_{\imath}} \Gamma\left(l_{H}, f\right)$ of the polar curve at $c_{\imath}$ (by our assumptions in Lemma 2.2). We deduce, see e.g. [Ti-4, Theorem 3.6], that if $\operatorname{dim}\left(\operatorname{Sing} F_{a}\right) \leq 0$, then:

$$
b_{n-2}\left(M_{H, r_{\imath}}\right)=\operatorname{int}_{c_{l}}\left(\Gamma\left(l_{H}, f\right), F_{a}\right)-\mu\left(F_{a}, c_{\imath}\right),
$$

where $\operatorname{int}_{c_{l}}\left(\Gamma\left(l_{H}, f\right), F_{a}\right)$ denotes the intersection multiplicity at $c_{\imath}$ and $\mu\left(F_{a}, c_{\imath}\right)$ is the Milnor number of the hypersurface singularity $\left(F_{a}, c_{\imath}\right)$. (If $F_{a}$ is nonsingular at $c_{\imath}$, then $\mu\left(F_{a}, c_{\imath}\right)=0$.)

After the second author's paper [Ti-4], there is the following homotopy model: $F_{a}$ is built from the generic hyperplane section $F_{a} \cap H$ to which one attaches $\gamma_{a}^{n-1}-\mu\left(F_{a}\right)$ cells of dimension $n-1$, where $\mu\left(F_{a}\right)$ is the sum of the Milnor numbers of the singularities of the hypersurface $F_{a}$ and $\gamma_{a}^{n-1}$ is the generic polar intersection multiplicity. We recall from [Ti-4] the definition of $\gamma_{a}^{n-1}$ and of the set of generic polar intersection multiplicities $\gamma_{a}^{*}:=$ $\left\{\gamma_{a}^{n-1}, \gamma_{a}^{n-2}, \ldots, \gamma_{a}^{1}, \gamma_{a}^{0}\right\}$ :

$$
\gamma_{a}^{n-1}=\operatorname{int}\left(\Gamma\left(l_{H}, f\right), F_{a}\right), \quad H \in \Omega_{a},
$$

where $\operatorname{int}\left(\Gamma\left(l_{H}, f\right), F_{a}\right)$ denotes the sum of the local intersection multiplicities at each point of the finite set $\Gamma\left(l_{H}, f\right) \cap F_{a}$. Next, take a hyperplane $\mathcal{H} \in \Omega_{a}$ and denote by $\gamma_{a}^{n-2}$ the generic polar intersection multiplicity at $a \in \mathbb{C}$ for the polynomial function $f_{\mid \mathcal{H}}: \mathbb{C}^{n-1} \rightarrow \mathbb{C}$, where $\mathcal{H}$ is identified to $\mathbb{C}^{n-1}$. By induction, we define in this way $\gamma_{a}^{n-1}$, for $1 \leq i \leq n-1$. By definition, $\gamma_{a}^{0}:=\operatorname{deg} f$.

By a standard connectivity argument, the set of polar intersection multiplicities is well-defined i.e., it does not depend on the choices of generic hyperplanes. 
The generic skeleton $\mathrm{Sk}_{a}(f)$ of $F_{a}$ is defined as the $C W$-complex obtained by successively attaching to $\gamma_{a}^{0}$ points $\gamma_{a}^{1}$ cells of dimension $1, \gamma_{a}^{2}$ cells of dimension $2, \ldots, \gamma_{a}^{n-2}$ cells of dimension $n-2$ and finally $\gamma_{a}^{n-1}-\mu\left(F_{a}\right)$ cells of dimension $n-1$.

By the above, the generic skeleton does not depend on the choices of generic hyperplanes. We refer to [Ti-4] for the details and an application of $\gamma_{a}^{*}$ to equisingularity at infinity. Computations of $\gamma^{*}$ can be found in the Examples at the end.

One of the consequences of the above model is the following:

Corollary 2.6. Let $H \in \Omega$. If $\Gamma\left(l_{H}, f\right) \cap F_{a}=\emptyset$ then $H_{\jmath}\left(F_{a}, \mathbb{Z}\right)=0$, for $j \geq n-1$.

\section{§3. A Global Geometric Monodromy}

We proceed to construct a geometric monodromy of a polynomial function by using the key lemma 2.1 and the generic skeleton $\mathrm{Sk}_{c}(f)$ of a typical fiber of $F_{c}$. Let $H \in \Omega$ and let $\tilde{\Lambda} \subset \Lambda_{H} \subset \mathbb{C}$ be the finite set $\tilde{\Lambda}:=p\left(\operatorname{Sing}_{\mathcal{W}} p\right)$, in the notations of 2.1. For any simple loop within $\mathbb{P}^{1} \backslash \tilde{\Lambda} \cup\{\infty\}$ we may define a geometric monodromy as follows. By the proof of Lemma 2.1, the map $p: \mathbb{X} \backslash p^{-1}(\tilde{\Lambda} \cup\{\infty\}) \rightarrow \mathbb{C} \backslash \tilde{\Lambda}$ is a locally trivial stratified fibration. In particular, the restriction of $p$ on the open stratum $\mathbb{C}^{n}$ is a $\mathrm{C}^{\infty}$ locally trivial fibration. As in the proof of the Thom-Mather Isotopy Lemma (see e.g. [Ve]) one can produce a trivializing vector field tangent to the strata at infinity. We therefore get a geometric monodromy representation

$$
\rho: \pi_{1}\left(\mathbb{P}^{1} \backslash \tilde{\Lambda} \cup\{\infty\}\right) \rightarrow \operatorname{Diff}(F),
$$

where $F$ is a general fiber of $f$ and $\operatorname{Diff}(F)$ is the group of $\mathrm{C}^{\infty}$-diffeomorphisms of $F$. We may replace $\tilde{\Lambda}$ by the set of atypical values $\Lambda$. This induces an algebraic monodromy representation $\rho_{\text {alg }}: \pi_{1}\left(\mathbb{P}^{1} \backslash \Lambda \cup\{\infty\}\right) \rightarrow H_{*}(F, \mathbb{Z})$.

\subsection{Monodromy at an atypical value and at infinity}

Some more structure on the trivializing vector field could allow to find results on monodromy. We therefore focus on defining a geometric monodromy of a general fiber of $f$ along a small circle in the base space $\mathbb{P}^{1} \backslash \Lambda \cup\{\infty\}$. We recall that $\Lambda_{H}=t\left(\mathbb{C}_{r} \times\left(\right.\right.$ Sing $\left.\left.{ }_{\mathcal{W}} p \cup A_{H}\right)\right)$ is a finite set containing $\Lambda$.

Take a small closed disc $D_{a}$ at $a \in \Lambda \cup\{\infty\}$ such that $\Lambda_{H} \cap D_{a}=\{a\}$. We assume that $H \in \Omega_{a}$. By Lemma 2.1, one can lift the unitary vector field $\mathbf{u}$ on the circle $\partial D_{a}$ to a vector field $\mathbf{w}$ in the tube $f^{-1}\left(\partial D_{a}\right)$ such that $\mathbf{w}$ is tangent 
to $t^{-1}\left(\partial D_{a}\right) \cap \mathbf{Y}^{\infty}$ in a stratified sense and tangent to $\Gamma\left(l_{H}, f\right) \cap f^{-1}\left(\partial D_{a}\right)$, for some general $H \in \Omega_{a}$. Note that the set $\Gamma\left(l_{H}, f\right) \cap f^{-1}\left(\partial D_{a}\right)$ is a finite union of circles.

Moreover, one can construct a vector field $\mathbf{w}$ by lifting $\mathbf{u}$ in two steps:

$$
f^{-1}\left(\partial D_{a}\right) \stackrel{(l, f)}{\longrightarrow} \mathbb{C} \times \partial D_{a} \stackrel{\mathrm{pr}_{2}}{\longrightarrow} \partial D_{a} .
$$

This idea was used in the local case by Lê D.T. [Lê-1], [Lê-2]. In the global setting, we may decompose the monodromy flow in regions where the local carrousel construction of Lê can be used. Let $\bar{\Gamma}\left(l_{H}, f\right)$ denote the closure of $\Gamma\left(l_{H}, f\right)$ in $\mathbb{X}$. There is a "carrousel" associated to each point $q \in \bar{\Gamma}\left(l_{H}, f\right) \cap$ $p^{-1}(a)$, including the case where $q \in \mathbb{X}^{\infty} \cap p^{-1}(a)$, as follows. We use the notations from 2.1. We have defined at (1) the space $\mathbf{Y} \subset \mathbb{C}_{r} \times \mathbb{P}^{n} \times \mathbb{C}_{t}$ with projection $(r, t): \mathbf{Y} \rightarrow \mathbb{C}_{r} \times \mathbb{C}_{t}$. We now consider the closure $\hat{\mathbf{Y}}_{r}$ of $\mathbf{Y}$ in $\mathbb{P}^{n} \times \mathbb{P}_{r}^{1} \times \mathbb{C}_{t}$ and denote by $\hat{r}: \hat{\mathbf{Y}}_{r} \rightarrow \mathbb{P}^{1}$ the projection which extends $r: \mathbf{Y} \rightarrow \mathbb{C}_{r}$. First we lift the vector field $\mathbf{u}$ by $\mathrm{pr}_{2}$ to a vector field $\mathbf{v}$ on $\mathbb{C}_{r} \times \partial D_{a}$ with the following properties. There is a small closed disc $\delta$ at each point $\hat{r}(q)$ of $\mathbb{P}^{1}, q \in \bar{\Gamma}\left(l_{H}, f\right) \cap t^{-1}(a)$, such that $\mathbf{v}$ is the carrousel vector field on $\delta \times \partial D_{a}$, for small enough $D_{a}$. In particular, the lift $\mathbf{v}$ of $\mathbf{u}$ to $\mathbb{C}_{r} \times \partial D_{a}$ is tangent to the discriminant $\Delta\left(l_{H}, f\right)$. By definition, the vector field $\mathbf{v}$ is the identical lift by the projection $\operatorname{pr}_{2}:\{b\} \times \partial D_{a} \rightarrow \partial D_{a}$, for any point $b \in \partial \delta$; moreover, this is the case for any point $b \in \mathbb{P}^{1} \backslash \cup_{\imath=1}^{k} \delta_{\imath}$, where $\delta_{\imath}$ is a small enough disc centered at $d_{\imath}$ and the set $\left\{d_{1}, \ldots, d_{k}\right\} \in \mathbb{P}^{1}$ is the image by $\hat{r}$ of the set $\bar{\Gamma}\left(l_{H}, f\right) \cap p^{-1}(a)$.

This special vector field $\mathbf{v}$ is now lifted to $f^{-1}\left(\partial D_{a}\right)$ via the stratified fibration (2) giving rise, by integration, to a geometric monodromy, denoted by $h_{a}$. Note that, for some point $b \in \mathbb{P}^{1} \backslash \cup_{\imath=1}^{k} \delta_{\imath}$, this monodromy restricts to a monodromy of the slice fibration:

$$
f^{-1}\left(\partial D_{a}\right) \cap l_{H}^{-1}(b) \rightarrow \partial D_{a} .
$$

The action of the monodromy $h_{a}$ on the pair $\left(F_{c}, F_{c} \cap l_{H}^{-1}(b)\right)$, where $c \in \partial D_{a}$, is called relative geometric monodromy and will be denoted by $h_{a}^{\text {rel }}$.

Note 3.2. Let $H \in \Omega_{a}$. If $q \in \bar{\Gamma}\left(l_{H}, f\right) \cap p^{-1}(a) \cap \mathbb{X}^{\infty}$ then $\hat{r}(q)=\infty \in$ $\mathbb{P}^{1}$.

\section{§4. Localization of Monodromy}

Localizing the change of topology from a typical fiber to an atypical one is a problem that cannot be solved for any polynomial $f: \mathbb{C}^{n} \rightarrow \mathbb{C}$, since its 
singularities at infinity (at least in the meaning of this paper. see bellow) may be non-isolated.

Definition 4.1. We say that $f$ has isolated $\mathcal{W}$-singularities at $a \in \mathbb{C}$ (or at $F_{a}$ ) if $\operatorname{dim} \operatorname{Sing}_{\mathcal{L}} p \cap p^{-1}(a)=0$.

Notice that isolated $\mathcal{W}$-singularities at $a \in \mathbb{C}$ implies that the hypcrsurface $F_{a}$ has isolated singularities. Under this isolatedness condition we irave proved a structure result for the general fibre.

Theorem $4.2[\mathrm{ST}]$. If $f: \mathbb{C}^{n} \rightarrow \mathbb{C}$ has isolated $\mathcal{W}$-singularities at all fibers then its general fibre is homotopy equivalent to a bouquet of spheres of dimension $n-1$. The number of spheres is equal to $\mu+\lambda$, where $\mu$ denotes the total Milnor number of the isolated singularities of $f$ and $\lambda$ denotes the total "Milnor number at infinity" of the isolated $\mathcal{W}$-singularities at infinity (defined in loc cit).

Following [Ti-3, Section 4], we say that the variation of topology of the fibers of $f$ at $a \in \mathbb{C}$ is localizable if there is a finite set $\left\{a_{1}, \ldots, a_{k}\right\} \in p^{-1}(a)$ such that the restriction $f_{1}:\left(\mathbb{C}^{n} \backslash \cup_{\imath=1}^{k} B_{\imath}\right) \cap f^{-1}\left(D_{a}\right) \rightarrow D_{a}$ is a trivial fibration, where $B_{\imath} \subset \mathbb{X}$ is a small enough ball centered at $a_{\imath}$. In this case, we also say that the monodromy $h_{a}$ is localizable at the points $a_{1}, \ldots, a_{k}$, in the sense that the geometric monodromy $h_{a}$ is trivial on the complement of the balls $F_{c} \backslash \cup_{\imath=1}^{k} B_{i}$.

Proposition 4.3 [Ti-3]. If $f$ has isolated $\mathcal{W}$-singularities at $a \in \mathbb{C}$ then the variation of topology of the fibers of $f$ at $a$ is localizable at the points of the finite set $p^{-1}(a) \cap \operatorname{Sing}{ }_{w} p$. In particular, the monodromy $h_{a}$ is localizable at the isolated $\mathcal{W}$-singularities.

Our scope is to show that certain localization results for the relat: $(1,1 \%$ : odromy $h_{a \text {,rel }}$ hold without any assumption on the $\mathcal{W}$-singularities of $f$.

\section{First localization of relative monodromy}

We shall keep using the notations in the previous section. Recall that $d_{1}, d_{2}, \ldots, d_{k}$ were the points where the closure $\bar{\Delta}\left(l_{H}, f\right) \subset \mathbb{P}_{r}^{1} \times \mathbb{C}_{t}$ of the discriminant $\Delta\left(l_{H}, f\right)$ cuts the projective line $\{t=a\} \subset \mathbb{P}_{r}^{1} \times \mathbb{C}_{t}$. We consider small discs $\delta_{i} \subset \mathbb{P}_{r}^{1}$ at $d_{i}$ and fix some points $s_{\imath} \in \partial \delta_{\imath}$, for $i \in\{1, \ldots k\}$. We denote $\check{\delta}_{i}:=\delta_{i}$ if $d_{i} \in \mathbb{C}_{r}$ and $\check{\delta}_{i}:=\delta_{i} \backslash \alpha_{i}$ if $d_{i}=\infty$, where $\alpha_{\imath}$ is the radius from $d_{i}$ to a point on the circle $\partial \delta_{i}$ different from $s_{\imath}$. 
By construction, $\check{\delta}_{\imath} \times\{c\}$ contains all the points of $\bar{\Delta}_{l}\left(l_{H}, f\right) \cap \mathbb{P}_{r}^{1} \times\{c\}$, where $c \in \partial D_{a}$ and $\bar{\Delta}_{\imath}\left(l_{H}, f\right)$ denotes the germ of $\bar{\Delta}\left(l_{H}, f\right)$ at $\left(d_{2}, a\right) \in \mathbb{P}^{1} \times \mathbb{C}$. The structure of the generic skeleton $\mathrm{Sk}_{c}(f)$ implies that the relative homology $H_{*}\left(F_{c}, F_{c} \cap l_{H}^{-1}(b)\right)$ is concentrated in dimension $n-1$, where $b \in \mathbb{P}^{1} \backslash \cup_{l=1}^{k} \delta_{l}$. We have the following.

Proposition 4.4. The relative homology splits into a direct sum:

$$
H_{n-1}\left(F_{c}, F_{c} \cap l_{H}^{-1}(b)\right)=\oplus_{l=1}^{k} H_{n-1}\left(F_{c} \cap l_{H}^{-1}\left(\check{\delta}_{\imath}\right), F_{c} \cap l_{H}^{-1}\left(s_{\iota}\right)\right)
$$

and the relative monodromy splits accordingly:

$$
\left(h_{a}^{\mathrm{rel}}\right)_{*}=\oplus_{1-1}^{k}\left(h_{a}^{\mathrm{rel}}\right)_{*, 2}
$$

where $\left(h_{a}^{\mathrm{rel}}\right)_{*, \imath}$ denotes the monodromy acting on $H_{n-1}\left(F_{c} \cap l_{H}^{-1}\left(\check{\delta}_{\imath}\right), F_{c} \cap l_{H}^{-1}\left(s_{\imath}\right)\right)$.

Proof. The geometric monodromy $h_{a}^{\text {rel }}$ constructed above acts on the exact sequence of the pair $\left(F_{c}, F_{c} \cap l_{H}^{-1}(b)\right)$. By an excision argument applied to the projection $l_{H}: F_{C} \rightarrow \mathbb{C}$, we get the homology splitting. Next, the geometric monodromy $h_{a}^{\text {rel }}$ acts on the pair of spaces $\left(F_{c} \cap l_{H}^{-1}\left(\check{\delta}_{\imath}\right), F_{c} \cap l_{H}^{-1}\left(s_{\imath}\right)\right)$ if and only if $d_{\imath} \in \mathbb{C}$. Nevertheless, in case the center $d_{l}$ is the point $\infty \in \mathbb{P}^{1}$. the algebraic monodromy still acts on the relative homology $H_{n-1}\left(F_{c} \cap l_{H}^{-1}\left(\check{\delta}_{\imath}\right), F_{c} \cap\right.$ $\left.l_{H}^{-1}\left(s_{\imath}\right)\right)$.

\section{Second localization of relative monodromy}

We further localize the relative monodromy in the source space. Let $a \in \mathbb{C}$. By Lemma 2.2, for $H \in \Omega_{a}$, the map $l_{H}: F_{a} \rightarrow \mathbb{C}$ has isolated critical points (in the stratified sense), which points are exactly the set $\Gamma\left(l_{H}, f\right) \cap F_{a}$ and has no singularities at infinity. It follows that the map $l_{H}: F_{C} \rightarrow \mathbb{C}$ has the same properties, for any $c \in D_{a}$, for small enough disc $D_{a}$. If $a$ is $x$ then the map $l_{H}: F_{c} \rightarrow \mathbb{C}$ also has these properties, for any $c \in \mathbb{C}$ within a small enough neighbourhood of $\infty \in \mathbb{P}^{1}$.

Let $\left\{p_{\imath, \jmath}\right\}_{j} \in l_{\text {, }}$ denote the set of points $\Gamma\left(l_{H}, f\right) \cap F_{c} \cap l_{H}^{-1}\left(\check{\tilde{\delta}}_{l}\right)$. Take Milnor data for the germs $l_{H}:\left(F_{c}, p_{\imath . \jmath}\right) \rightarrow \mathbb{C}$. which means: small enough discs $D_{\imath . \jmath} \subset$ $\check{\delta}_{\iota}$ centered at $l_{H}\left(p_{\imath, \jmath}\right)$ and small balls $B_{\imath, \jmath} \subset F_{c} \cap l_{H}^{-1}\left(\check{\delta}_{\imath}\right)$ centered at $p_{\imath, \jmath}$. We assume that $D_{\imath \cdot \jmath_{1}}=D_{\imath \cdot \jmath_{2}}$ if $l_{H}\left(p_{\imath \cdot \jmath_{1}}\right)=l_{H}\left(p_{\imath, J_{2}}\right)$. Note again that, by 2.1 and 2.2 , these are germs of complex Morse singularities. We get the following result:

Proposition 4.5. Let $a \in \mathbb{P}^{1}$ and let $i$ be fixed. The homology of the pair $\left(F_{c} \cap l_{H}^{-1}\left(\check{\delta}_{\imath}\right), F_{c} \cap l_{H}^{-1}\left(s_{\imath}\right)\right)$ splits into a direct sum

$$
\oplus_{\jmath} \in l_{1} H_{n-1}\left(F_{c} \cap l_{H}^{-1}\left(D_{\imath, \jmath}\right) \cap B_{\imath, \jmath}, F_{c} \cap l_{H}^{-1}\left(s_{\imath, \jmath}\right) \cap B_{\imath, \jmath}\right),
$$


where $s_{\imath . j} \in \partial D_{\imath, j}$ are some fixed points. The restriction of the relative monodromy $h_{a, \text { rel }}$ acts on the set of all pairs $\left(F_{c} \cap l_{H}^{-1}\left(D_{i, \jmath}\right) \cap B_{\imath, \jmath}, F_{c} \cap l_{H}^{-1}\left(s_{\imath, \jmath}\right) \cap B_{i, \jmath}\right)$ by permutations, via diffeomorphisms (i.e. $h_{a \text {.rel }}$ sends a pair to another one. diffeomorphically).

Proof. First we get by excision the following splitting:

$$
H_{n-1}\left(F_{c} \cap l_{H}^{-1}\left(\check{\delta}_{\imath}\right), F_{c} \cap l_{H}^{-1}\left(s_{\imath}\right)\right)=\oplus H_{n-1}\left(F_{c} \cap l_{H}^{-1}\left(D_{\imath, \jmath}\right), F_{c} \cap l_{H}^{-1}\left(s_{\imath, \jmath}\right)\right),
$$

where the sum is taken over the distinct discs $D_{\imath, \jmath}$, with fixed $i$. Next, by the above arguments, the map:

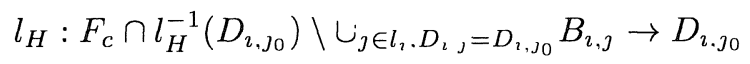

is a trivial fibration, for any $j_{0} \in l_{\imath}$. It is indeed so since this map has no singularities at infinity and its fibers are transverse to the spheres $\partial B_{\imath, \jmath}$. The splitting of homology follows. The second statement is a consequence of the fact that our vector field is tangent to the polar curve.

\section{Remarks 4.6 .}

(a) In general, the relative monodromy does not split following the direct sum of Proposition 4.5. There are interactions among the relative cycles which depend on the global carrousel motion and also on the action of $h_{a}$ on the fiber $F_{c} \cap l_{H}^{-1}\left(s_{\imath, \jmath}\right)$. Moreover, it may happen that one or more branches of the discriminant locus at $d_{\imath}$ are multiple, since being the image of more than one branches of the polar curve.

(b) Let $H \in \Omega_{a}$. Let $p \in F_{a} \cap \Gamma\left(l_{H}, f\right)$ such that $p \notin \operatorname{Sing} F_{a}$. By Lemma 2.2, since $p$ is a Morse point of the map $l_{H \mid F_{a}}$, the polar curve is nonsingular at $p$ and it cuts the nearby fiber $F_{c}$ at a single point $p_{\imath, 1}$ within a small neighborhood of $p$. Then the relative monodromy acts on the pair $\left(F_{c} \cap\right.$ $\left.l_{H}^{-1}\left(D_{\imath .1}\right) \cap B_{\imath, 1}, F_{c} \cap l_{H}^{-1}\left(s_{\imath, 1}\right) \cap B_{\imath .1}\right)$ as the identity. Indeed, the maps $f_{\mid}: f^{-1}(D) \cap l_{H}^{-1}\left(s_{\imath, 1}\right) \cap B_{\imath, 1} \rightarrow D$ and $f_{\mid}: f^{-1}(D) \cap l_{H}^{-1}\left(D_{\imath, 1}\right) \cap B_{\imath, 1} \rightarrow D$ are trivial stratified fibrations for small enough $D_{\imath .1}$, the strata being $\Gamma\left(l_{H}, f\right)$ and its complement.

When $\delta_{\imath} \subset \mathbb{C}^{n}$, the relative monodromy $\left(h_{a}^{\text {rel }}\right)_{*, \imath}$ from Proposition 4.4 can moreover be localized at the points of intersection $\Gamma\left(l_{H}, f\right) \cap F_{a} \cap l_{H}^{-1}\left(\delta_{\imath}\right)=$ $\left\{b_{1}, \ldots, b_{g_{2}}\right\}$. 
Proposition 4.7. Let $\delta_{\imath} \subset \mathbb{C}^{n}$, for some $i \in\{1, \ldots, k\}$. Then there is the following splitting:

$$
\left(h_{a}^{\mathrm{rel}}\right)_{*, \imath}=\oplus_{\jmath=1}^{g_{\imath}}\left(h_{b_{\jmath}}^{\mathrm{rel}}\right)_{*},
$$

where $\left(h_{b_{\jmath}}^{\mathrm{rel}}\right)_{*}$ denotes the local relative monodromy of the germ $f_{\mid}:\left(\mathbb{C}^{n}, b_{\jmath}\right) \rightarrow \mathbb{C}$ with respect to the linear function $l_{H}$.

Proof. Since the map $l_{H}: F_{c} \cap l_{H}^{-1}\left(\delta_{\imath}\right) \rightarrow \delta_{\imath}$ is trivial at infinity, for all $c$ close enough to $a$, we may excise the exterior of some small enough polydiscs $P_{\jmath}:=B_{\jmath} \times \delta_{\imath}$ centered at $b_{\jmath}$, for $j \in\left\{1, \ldots, g_{\imath}\right\}$, and get the splitting:

$$
\begin{aligned}
& H_{n-1}\left(F_{c} \cap l_{H}^{-1}\left(\delta_{\imath}\right), F_{c} \cap l_{H}^{-1}\left(s_{\imath}\right)\right) \\
& \quad=\bigoplus_{j=1}^{g_{\imath}} H_{n-1}\left(P_{\jmath} \cap F_{c} \cap l_{H}^{-1}\left(\delta_{\imath}\right), P_{\jmath} \cap F_{c} \cap l_{H}^{-1}\left(s_{\imath}\right)\right) .
\end{aligned}
$$

The monodromy splits accordingly. The right hand terms are just the local relative homology groups of the germs $f_{\mid}:\left(\mathbb{C}^{n}, b_{\jmath}\right) \rightarrow \mathbb{C}$ with respect to the linear function $l_{H}$.

Proposition 4.8. Let $H \in \Omega_{a}$. If $f$ has isolated $\mathcal{W}$-singularities at a (see Definition 4.1), then the restriction $h_{a j H_{r}}$ of $h_{a}$ to a general hyperplane $H_{r}:=\left\{l_{H}=r\right\}$ can be chosen to be the identity.

Proof. The second author has proved in [Ti-3, §5] that, if $H \in \Omega$, then the Whitney stratification $\mathcal{W}$ attached to $f$ induces canonically a Whitney stratification $\mathcal{W}_{H_{r}}$ attached to the restriction $f_{\mid H_{r}}$. Then one proves, see [Ti-3, Lemma 5.4], that $\operatorname{dim} \operatorname{Sing} \mathcal{W}_{H_{r}} p^{\prime} \leq \operatorname{dim} \operatorname{Sing}{ }_{\mathcal{W}} p-1$, where $p^{\prime}$ is the extension of $f_{\mid H_{r}}$ (as $p$ is the one of $f$ defined in $\S 2$ ), under the condition: $\operatorname{dim} \operatorname{Sing}{ }_{\mathcal{W}} p \geq 1$. It turns out that, by choosing $H \in \Omega_{a}$, one can easily drop this condition completely when referring to only the fibre over $a$. The same proof works with minor changes, so we skip it:

Lemma (cf. [Ti-3]). If $H \in \Omega_{a}$ then

$$
\operatorname{dim} \operatorname{Sing} \mathcal{W}_{H_{r}} p^{\prime} \cap p^{\prime-1}(a) \leq \operatorname{dim} \operatorname{Sing}{ }_{\mathcal{W}} p \cap p^{-1}(a)-1 \text {. }
$$

Now, if $\operatorname{dim} \operatorname{Sing}{ }_{\mathcal{W}} p=0$, then it follows from this lemma that $f_{\mid H_{r}}$ has no $\mathcal{W}$-singularities on the fibre over $a$, hence this fibre is typical for $f_{\mid H_{r}}$ and therefore the monodromy of $f_{\mid H_{r}}$ around $a$ is isotopic to the identity. 


\section{§5. Zeta Function of Monodromy Via the Relative Monodromy}

The relative monodromy is part of the monodromy of $f$ around an atypical value $a \in \mathbb{P}^{1}$. We show the precise meaning of this at the zeta function level. Let us first give the definition we work with.

Definition 5.1. Let $h_{*}$ be the algebraic monodromy associated to a fibration over a circle $S^{1}$ and let $H_{\imath}$ denote the $i^{\text {th }}$ homology group, over $\mathbb{C}$, of the fibre. One calls zeta function of $h_{*}$ the following rational function:

$$
\dot{\zeta}_{h_{\star}}(t)=\prod_{i \geq 0} \operatorname{det}\left[\mathrm{id}-t h_{*}: H_{\imath} \rightarrow H_{\imath}\right]^{(-1)^{\imath+1}}
$$

As a consequence of the localization of the relative monodromy, we prove the following general zeta function formula for the monodromy $\left(h_{a}\right)_{*}$, with no restrictions on $f$.

Theorem 5.2. Let $f: \mathbb{C}^{n} \rightarrow \mathbb{C}$ be any polynomial function. Let $a \in \mathbb{P}^{1}$ and let $H \subset \Omega_{a}$. Then

$$
\zeta_{h_{a}}(t)=\zeta_{h_{a \mid F^{\prime}}}(t) \cdot \prod_{i=1}^{k} \zeta_{\mathrm{rel}, \imath}(t)
$$

where $F^{\prime}$ is a general fiber of the map $\left(l_{H}, f\right)$ and by $\zeta_{\text {rel }, l}(t)$ we denote the zeta-function of the relative monodromy $\left(h_{a}^{\mathrm{rel}}\right)_{*, \imath}$. The index $i$ runs over the points $\bar{\Delta}\left(l_{H}, f\right) \cap\{t=a\}$. For $d_{i}=\infty$ we have:

$$
\zeta_{\mathrm{rel}, \imath}(t)=\zeta_{\delta^{*}}(t)
$$

where $\zeta_{\delta^{*}}$ is the zeta function of the monodromy acting on the space $F_{c} \cap l_{H}^{-1}\left(\delta_{\imath}^{*}\right)$, with $\delta^{*}:=\delta_{\imath}^{*}$ denoting the pointed disc centered at $\infty \in \mathbb{P}^{1}$.

Proof. The monodromy acts on the homology sequence of the pair $\left(F_{c}, F_{c} \cap\right.$ $\left.l_{H}^{-1}(b)\right)$. We get the 4-term exact sequence:

$0 \rightarrow H_{n-1}\left(F_{c}\right) \rightarrow H_{n-1}\left(F_{c}, F_{c} \cap l_{H}^{-1}(b)\right) \rightarrow H_{n-2}\left(F_{c} \cap l_{H}^{-1}(b)\right) \rightarrow H_{n-2}\left(F_{c}\right) \rightarrow 0$

and, for $j \geq 3$, the isomorphisms:

$$
0 \rightarrow H_{n-\jmath}\left(F_{c} \cap l_{H}^{-1}(b)\right) \rightarrow H_{n-\jmath}\left(F_{c}\right) \rightarrow 0 .
$$

By Proposition 4.4 the action of $h_{a}^{\text {rel }}$ splits and therefore the zeta-function decomposition follows. To prove the last assertion, we start by decomposing 
$\mathbb{C}$ into the union of a big disc $D$ with the pointed disc at infinity $\delta^{*}$, their intersection being a thickened circle $A$. A Mayer-Vietoris argument shows that $\zeta_{h_{a}}(t)=\zeta_{D}(t) \cdot \zeta_{\delta^{*}}(t)$. Next, $D$ retracts to the union of the discs $\delta_{\imath} \subset \mathbb{C}$ together with simple paths connecting all those to one exterior point $p \in \mathbb{C}$. Excising now $l_{H}^{-1}(\gamma)$ in the relative homology, we get the formula:

$$
\zeta_{h_{a}}(t)=\zeta_{h_{a \mid F^{\prime}}}(t) \cdot \prod_{d_{\imath} \subset \mathbb{C}} \zeta_{\mathrm{rel} . \imath}(t) \cdot \zeta_{\delta^{*}}(t)
$$

Comparing to the previous formula for $\zeta_{h_{a}}$, we get the claimed relation.

Zeta function formulae have been proven for special classes of polynomials $f:$ in terms of Newton polyhedra-under nondegeneracy conditions [LS], in terms of the projective compactification of $f$-when this is non-singular [GN]. In a recent preprint [GLM], a general zeta function formula is presented, using the fact that the zeta function is a constructible function.

Let us now focus on isolated $\mathcal{W}$-singularities case. Proposition 4.3 tells that, if $f$ has isolated $\mathcal{W}$-singularities at the fiber $F_{a}$ then the variation of topology is localizable and the monodromy $h_{a}$ is splitting. To make a precise statement about zeta function, let $\left\{q_{s} \mid s=1, \ldots, \nu\right\} \subset \bar{F}_{a}$ be the $\mathcal{W}$ singularities of $f$ at $F_{a}$. According to [Ti-3, 4.3], at each point $q_{s}$ there is a complete system of ball neighborhoods $B_{s . \varepsilon}$ within the space $\mathbb{X}$, such that the map

$$
f_{\uparrow}:\left(\mathbb{C}^{n} \backslash \cup_{s} \bar{B}_{s . \varepsilon}\right) \cap f^{-1}\left(D_{\delta}\right) \rightarrow D_{\delta}
$$

is a trivial fibration, for small enough $\varepsilon>0,0<\delta<<\varepsilon$ and $D_{\delta}$ is centered at a. Moreover, for any $s$, the restriction

$$
f_{\mid}: \mathbb{C}^{n} \cap B_{s, \varepsilon} \cap f^{-1}\left(D_{\delta} \backslash\{a\}\right) \rightarrow D_{\delta} \backslash\{a\}
$$

is locally trivial, cf. [Ti-3, 4.4]. Let us denote by $\zeta_{h_{a} . s}(t)$ the zeta function of the monodromy induced by the local fibration (6) on the pair $\left(\mathbb{C}^{n} \cap B_{s, \varepsilon} \cap f^{-1}\left(D_{\delta} \backslash\right.\right.$ $\left.\{a\}, F_{c} \cap B_{s, \varepsilon}\right)$, where $c \in \partial D_{\delta}$. We then have the following decomposition into local zeta functions:

Proposition 5.3. If $f$ has isolated $\mathcal{W}$-singularities at $a \in \mathbb{C}$. then:

$$
\zeta_{h_{a}}(t)=(1-t)^{-\chi\left(F_{a}\right)} \prod_{s=1}^{\nu} \zeta_{h_{a} . s}^{-1}(t),
$$

where $q_{1}, \ldots, q_{\nu}$ are the isolated singularities on $F_{a}$ or at infinity. 
Proof. From the homology exact sequence of the pair $\left(F_{D_{\delta}}, F_{c}\right)$ we get that $\zeta_{h_{a}}(t)=\zeta_{F_{D_{\delta}}}(t) \cdot \zeta_{\left(F_{D_{\delta}}, F_{c}\right)}^{-1}(t)$. Since by excision we have the direct sum decomposition:

$$
H_{\bullet}\left(F_{D_{\delta}}, F_{c}\right) \simeq \oplus_{s} H_{\bullet}\left(F_{D_{\delta}} \cap B_{s, \varepsilon}, F_{c} \cap B_{s, \varepsilon}\right),
$$

we get $\zeta_{\left(F_{D_{\delta}}, F_{c}\right)}^{-1}(t)=\prod_{s=1}^{\nu} \zeta_{h_{a}, s}^{-1}(t)$. Now, since the monodromy acts on $F_{D_{\delta}}$ as the identity and since $\chi\left(F_{D_{\delta}}\right)=\chi\left(F_{a}\right)$, we get our formula. Note that the relative homology $H_{\bullet}\left(F_{D_{\delta}}, F_{c}\right)$ is concentrated in dimension $n-1$, [ST].

\section{Remarks 5.4.}

(a) The above result holds in particular for polynomial functions $f: \mathbb{C}^{2} \rightarrow \mathbb{C}$, at any reduced fiber $F_{a}$. See the Examples.

(b) If $f$ has isolated $\mathcal{W}$-singularities at all fibers, then it follows from [ST, 3.5] that $\chi\left(F_{a}\right)=1+(-1)^{n-1}\left(\mu+\lambda-\mu_{a}-\lambda_{a}\right)$, where $\mu$, resp. $\lambda$, denote the total Milnor number, resp. the total Milnor number at infinity, of $f$ whereas $\mu_{a}$, resp. $\lambda_{a}$, is the sum of the Milnor numbers of the singularities of $F_{a}$. resp. the sum of the Milnor numbers at infinity of $F_{a}$ (as defined by Hà and Lê [HL] in case $n=2$ and by the authors in general [ST]).

(c) For those isolated singularity $q_{s} \in F_{a}$ (not at infinity), the space $F_{D_{\delta}} \cap B_{s, \varepsilon}$ is contractible and therefore we may replace in the formula $(7)$ the factor $\zeta_{h_{a}, s}^{-1}(t)$ by $(1-t) \zeta_{q_{s}}(t)$, where $\zeta_{q_{s}}(t)$ is the zeta function of the germ of $f$ at $q_{s}$.

(d) A'Campo's local result $\left[\mathrm{A}^{\prime} \mathrm{C}\right]$ on the Lefschetz number says that the Lefschetz number of a singular holomorphic germ is zero. As a consequence of formula (7), there is the following generalization to the global setting, in case of an $f$ with isolated $\mathcal{W}$-singularities:

$$
L\left(h_{a}\right)=\chi\left(F_{a} \backslash \operatorname{Sing} f\right)+\sum L_{s}^{\infty}\left(h_{a}\right)
$$

where $L_{s}^{\infty}\left(h_{a}\right)$ denotes the Lefschetz number of the monodromy acting on the pair $\left(\mathbb{C}^{n} \cap B_{s, \varepsilon} \cap f^{-1}\left(D_{\delta} \backslash\{a\}\right), F_{c} \cap B_{s, \varepsilon}\right)$ and the sum is taken over those $s$ such that the point $q_{s}$ is at infinity.

Section 6 contains several examples where $f$ has a singularity at infinity but $L^{\infty}$ is non-zero (unlike in the local case). 


\section{§6. Computing the Relative Monodromy at Infinity}

To compute $\zeta_{\text {rel, },}(t)$ and $\zeta_{\delta^{*}}(t)$ we may use the method developed by the second author in [Ti-1] based on a Mayer-Vietoris argument for an annular decomposition of the carrousel disc. This works reasonably well for a carrousel with only first order smaller carrousels (i.e. when the branches of the germs of $\bar{\Delta}$ have only one Puiseux pair), but computations are very hard in general. We refer for the theoretical treatment to [Ti-1].

We show in the following examples what is the influence of the vicinity of infinity upon relative monodromy. This in turn influences the (absolute) monodromy at an atypical value. We consider first the case of an isolated singularity at infinity with $\lambda=1$ : the local relative monodromy action on the unique relative cycle can only be $+\mathrm{id}$ or $-\mathrm{id}$. When dimension is fixed, both cases are possible and we show this by the first two examples $(n=2)$. This behavior is new: in the local case, the monodromy of the unique $(n-1)$-cycle of a Morse singularity only depends on the dimension; for $n=2$, it is the identity.

Example 6.1. $\quad f: \mathbb{C}^{2} \rightarrow \mathbb{C}, f(x, y)=x+x^{2} y$.

This came into attention due to Broughton $[\mathrm{Br}]$, as the simplest polynomial with a noncritical atypical fiber (see also [ST], [Ti-3] for further comments on it). The point $[0: 1] \in \mathbb{P}^{1}$ is a $\mathcal{W}$-isolated singularity at infinity for the fiber $F_{0}$, with $\lambda=1$.

For a general $l$, say $l=x+y$, the polar curve $\Gamma(l, f)$ intersects a general fiber $F_{\text {gen }}$ in 3 points and the fiber $F_{0}$ in 2 points, transversely. We get $\gamma_{\text {gen }}^{1}=3$, $\gamma_{\text {gen }}^{0}=\gamma_{0}^{0}=3$ and $\gamma_{0}^{1}=2$, therefore $\chi\left(F_{\text {gen }}\right)=0, \lambda\left(F_{0}\right)=1$ (which is of course clear, since $F_{\text {gen }} \stackrel{\text { ht }}{\simeq} S^{1}$ and $\left.F_{0} \stackrel{\text { ht }}{\simeq} \mathbb{C} \coprod S^{1}\right)$. The zeta-function of the monodromy $h_{0}$ around the value 0 is equal, by Proposition 4.8 and Theorem 5.2 , to $\zeta_{F^{\prime}}(t) \cdot \prod_{r=1}^{3} \zeta_{\text {rel, }, ~}(t)$. Let $\delta:=\delta_{1}$ be the disc centered at $\infty \in \mathbb{P}^{1}$ and let $\delta_{2}, \delta_{3}$ be the other two discs in $\mathbb{C}$. Then, by Remark 4.6(b), $\zeta_{\text {rel,2 }}(t)=$ $\zeta_{\text {rel }, 3}(t)=(1-t)$. To get $\zeta_{\text {rel,1 }}(t)$ we use Theorem 5.2 and compute $\zeta^{*}(t)$ instead, as follows.

We use the setting and notations of Proposition 4.5. The carrousel $\delta^{*}$ centered at $\infty \in \mathbb{P}^{1}$ contains only one little disc $D$ centered at the unique point $p \in \Delta \cap \delta^{*}$. Let $s \in \partial D$. Then $\zeta_{\delta^{*}}(t)$ is equal to $\zeta_{D, s}(t)$, the zeta function of the carrousel monodromy acting on $H_{1}\left(F_{c} \cap l_{H}^{-1}(D) \cap B, F_{c} \cap l_{H}^{-1}(s) \cap B\right) \simeq H_{1}(I, \partial I)$. Now the carrousel monodromy of the space $F_{c} \cap l_{H}^{-1}(s) \cap B$ is isotopic to the monodromy of this space along a loop around both the center of $\delta^{*}$ and the center of $D$. We decompose it into a simple loop around $\infty \in \delta$ followed by a small loop around $p$. Since $p$ is an $A_{1}$-singularity of $l_{H}$ restricted to $F_{c}$, the $l_{H^{-}}$ 
monodromy of $H_{1}(I, \partial I)$ around it is -id, since $\operatorname{dim}_{\mathbb{C}} F_{c}=1$. The monodromy around the point $\infty$ is also -id, by the following reason: the compactified curve $\bar{F}_{c}$ has an $A_{2}$ singularity at $[0: 1]$, if $c \neq 0$ (and an $A_{3}$ singularity if $c=0$ ). The loop in $F_{c}$ around this point is nothing else but the complex link monodromy of the germ of $\bar{F}_{c}$ (i.e. the monodromy of a general linear function on $\bar{F}_{c}$ ). We refer to $[\mathrm{GM}]$ for definitions and basic results about complex link. By a straightforward computation one finds:

Lemma. The complex link monodromy of the germ of a $A_{k}$-type curve singularity is $(-1)^{k-1} \mathrm{id}$.

It then follows that $\zeta_{\mathrm{rel}, 1}(t)=(1-t)$. Therefore $\zeta_{h_{0}}(t)=1$, since $\zeta_{h_{0 \mid F^{\prime}}}(t)=$ $(1-t)^{-3}$. The monodromy is itself the identity.

Example 6.2. $\quad f: \mathbb{C}^{2} \rightarrow \mathbb{C}, f(x, y)=x^{2} y^{2}+x y+x$.

This is contained in the classification list of polynomials of small degrees, with respect to their singularities including those at infinity, of the first author with Smeltink [SS]. There is a Morse singularity at $(0,-1)$, on the fiber $F_{0}$ and a singularity at infinity at $[0: 1] \in \mathbb{P}^{1}$ for the fiber $F_{-\frac{1}{1}}$. Hence $\mu=1$ and $\lambda=1$. The general fiber is homotopy equivalent to $S^{1} \vee S^{1}$. We may take $l=x+y$ as general linear form. Then $\Gamma(l, f)=\left\{2 x y^{2}+y+1-2 x^{2} y-x=0\right\}$ and its intersection with $F_{t}$ is 4 points, if $t=0$ or $t=-\frac{1}{4}$ and 5 points for the other values of $t$. The fiber $F^{\prime}=F_{\text {gen }} \cap\{x+y=s\}$ is 4 points, for generic $s$. We get $\gamma_{\text {gen }}^{1}=\gamma_{0}^{1}=5$ and $\gamma_{-\frac{1}{4}}^{1}=4$. We now compute the zeta-function of the monodromy $h_{-\frac{1}{4}}$. By the localization result Proposition 5.3. this is equal to $\zeta_{\delta^{*}}$, since the other contributions are trivial. Now, in the carrousel disc $\delta^{*}$ centered at $q_{1}=\bar{\Gamma}(l, f) \cap \bar{F}_{-\frac{1}{4}}$, the situation is similar to that in the first example. The only difference is that the complex link monodromy in cause is this time the one of an $A_{3}$ curve singularity (instead of a $A_{2}$ singularity). Using the above Lemma, this shows that our monodromy is the composition (-id) $\circ(-$ id). Finally, by Theorem 5.2, we get:

$$
\zeta_{h_{-\frac{1}{4}}}(t)=(1-t)^{-4}(1-t)^{4}(1+t)=1+t .
$$

We may deduce that the monodromy $h_{-\frac{1}{4}}$ acts on a certain basis of absolute cycles by switching those.

The zeta function $\zeta_{h_{0}}$ is easier to compute since it is localizable at the Morse singularity $(0,-1)$ and the local monodromy is the identity (acting on the local cycle $S^{1}$ ). Therefore, by Proposition 5.3 and the remarks following it:

$$
\zeta_{h_{0}}(t)=(1-t)^{-\chi\left(F_{a}\right)}(1-t) \zeta_{(0,-1)}(t)=1-t
$$


since $F_{a} \stackrel{\text { ht }}{\simeq} S^{1}$ and $\zeta_{(0 .-1)}(t)=1$. By using the next Remark and Example, we find the zeta function at infinity: $\zeta_{h_{x}}(t)=1-t^{2}$.

Remark 6.3. The family of polynomial functions $f_{d}=x+d x y+x^{2} y^{2}$ is a $(\mu+\lambda)$-constant family (for $d=0$ we have $\lambda=2$ and $\mu=0$ ). For such a family the monodromy fibration at $\infty \in \mathbb{P}^{1}$ is constant for $d \in \mathbb{C}$, by [Ti-2]. Nevertheless, the monodromy group (i.e. the group generated by loops around atypical values) is not the same. For $d \neq 0$, it is the example above. We shall investigate the case $d=0$ in the next, within a whole series.

Example 6.4. Series $E_{a . b}: g_{a . b}=x+x^{a+1} y^{b}, a \geq 1, b \geq 0$.

Let $a, b$ be fixed. There are no singularities in the affine, $\mu=0$. We may consider $y$ as general linear function, $y \in \Omega_{0}$, even though it is not a "most generic" one. Then, for generic $s$, the intersection $F_{t} \cap H_{s}$ consists of $(a+1)$ points, for any $t$. The intersections with the polar curve $\Gamma\left(y, g_{a . b}\right)$ are $F_{t} \cap \Gamma=b$ points, for $t \neq 0$ and $F_{0} \cap \Gamma=\emptyset$. Hence $\lambda=b$ and there is a single $\mathcal{W}$-singularity at the point at infinity $[0: 1] \in \mathbb{P}^{1}$. corresponding to the fibre $F_{0}$. The carrousel at infinity is governed by the polar image $\bar{\Delta}\left(y, g_{a . b}\right)=\left\{f^{a}=h z^{b}\right\} \subset \mathbb{C}^{2}$, where $z=1 / y$ and $K$ is a constant. Let $c=\operatorname{gcd}(a, b)$. The effect of a $2 \pi$ turn in $f$ is a $\frac{a}{b} 2 \pi$ turn in $\approx$. Therefore we have $b$ points in the carrousel disc $\delta^{*}$ and the carrousel monodromy cyclically permutes the points within $c$ cycles of length $b / c$. Hence for each cycle we find the relative monodromy matrix:

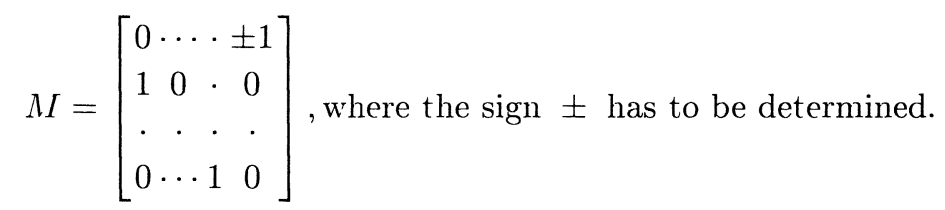

The total relative monodromy matrix is a block-matrix having on the diagonal $c$ matrices of type $M$ (and 0 in the rest). The \pm sign is determined by studying a $\frac{a}{c} 2 \pi$ monodromy in $z$ of one relative vanishing cycle, hence of its boundary. We embed the boundary cycle in the full slice $z=1 / y=$ const. in $F_{t}(t \neq 0)$. The $z$-monodromy on $F_{t}(t \neq 0)$ is isotopic to the $z$-monodromy of $F_{0}$, hence we study the complex link monodromy of $\mathbb{X}_{0}:=\mathbb{X} \cap p^{-1}(0)$ which has local equation $\left\{x z^{a+b}+x^{a+1}=0\right\}$. The slice $z=$ const. consists of $(a+1)$ points: $x=0$ and the solutions of $x^{a}=z^{a+b}$. On $x=0$, the $z$-monodromy works as the identity. On the other points, the effect of a $2 \pi$ turn in $z$ is a $\frac{b}{a} 2 \pi$ turn in $x$. Now we have to take the $a / c$ power of this, which is a $\frac{b}{c} 2 \pi$ turn in $x$, hence the identity! The sign determination is complete: it is +1 . For the zeta function, we get: $\zeta_{\delta^{*}}=\left(1-t^{\frac{b}{c}}\right)^{c}$. This coincides, by Theorem 5.2 , with the zeta function of $h_{a}$ and also with the one of $h_{\infty}$ (since $\left.\Lambda=\{0\}\right)$. 


\section{References}

[A'C] A'Campo, N., Le nombre de Lefschetz d'une monodromie, Nederl. Akad. Wet. Proc., Ser. A, 76 (1973), 113-118.

[ACD] Artal-Bartolo, E., Cassou-Nogués, P. and Dimca, A., Sur la topologie des polynomes complexes, in: eds. Arnold, V. I. et al., "Singularities. The Brieskorn Anniversary Volume", Prog. Math. 162, Birkhaeuser 1998, p. 317-343.

[Br] Broughton, S. A., On the topology of polynomial hypersurfaces, Proc. Symp. Pure. Math., 40, I (1983), 165-178.

[GLM] Gusein-Zade, S., Luengo, I. and Melle, A., On the zeta function of a polynomial at infinity, Preprint 1998, ESN preprint server http://www.mi.aau.dk/ esn.

[GM] Goresky, M. and MacPherson, R., Stratıfied Morse Theory, Springer-Verlag Berlin Heidelberg New-York, 1987.

[GN] Garcia Lopez, R. and Nemethi, A., On the monodromy at infinity of a polynomial map, Compositıo Math., 100, 2 (1996), 205-231.

[Ha] Hà, H. V., La formule de Picard-Lefschetz affine, C. R. Acad. Scı. Parıs, Ser. I, 321, 6 (1995), 747-750.

[HL] Hà, H. V. and Lê D. T., Sur la topologie des polynômes complexes, Acta Math. Vietnam., 9 (1984), 21-32.

[HMS] Henry, J. P., Merle, M. and Sabbah, C., Sur la condition de Thom stricte pour un morphisme analytique complexe, Ann. Scı. Ec. Norm. Sup. $4^{e}$ sérıe, 17 (1984), 227-268.

[Lê-1] Lê, D. T., La monodromie n'a pas de points fixes, J. Fac. Sc. Unıv. Tokyo, Sec. 1A, 22 (1975), 409-427.

[Lê-2] - The geometry of the monodromy theorem, C. P. Ramanujam - a tribute, Tata Institute, Springer-Verlag. 1978, p. 157-173.

[Lê-3] — Complex Analytic Functions with Isolated Singularities, J. Algebraıc Geom., 1 (1992), 83-100.

[LW] Lê, D. T. and Weber, C., A geometrical approach to the Jacobian conjecture for $n=2$, Kodaı Math. J., 17, 3 (1994), 374-381.

[LS] Libgober, A. and Sperber, S., On the zeta function of monodromy of a polynomial map, Compositıo. Math., 95, 3 (1995), 287-307.

[MW] Michel, F. and Weber, C., On the monodromies of a polynomial map from $\mathbb{C}^{2}$ to $\mathbb{C}$, manuscript 1998.

[Sa] Sabbah, C., Monodromy at Infinity and Fourier Transform, Publ. RIMS, Kyoto Unıv., 33 (1997), 643-685.

[SS] Siersma, D. and Smeltink, J., Classification of singularities at infinity of polynomials of degree 4 in two variables, Preprınt no. 945 (1996), University of Utrecht, Georgıan Math. J., 7, 1 (2000), 179-190.

[ST] Siersma, D. and Tibăr, M., Singularities at infinity and their vanishing cycles, Duke Math. J., 80:3 (1995), 771-783.

[ST-2] - Topology of polynomial functions and monodromy dynamics, C. R. Acad. Scı. Parıs, Sérıe I, 327 (1998), 655-660.

[Te] Teissier, B., Varietés polaires 2: Multiplicités polaires, sections planes et conditions de Whitney, Géométrie Algèbrique à la Rabida, Sprınger L.N.M., 961, 314-491.

[T] Thom, R., Ensembles et morphismes stratifiés, Bull. Amer. Math. Soc., 75 (1969), 249-312.

[Ti-1] Tibăr. M., Carrousel monodromy and Lefschetz number of singularities, Ensergn. Math., 39 (1993), 233-247.

[Ti-2] - On the monodromy fibration of polynomial functions with singularities at infinity, C. R. Acad. Scı., Parıs, Ser. I, 324, 9 (1997), 1031-1035.

[Ti-3] - Topology at infinity of polynomial maps and Thom regularity condition, Composıtıo Math.. 111, 1 (1998), 89-109.

[Ti-4] - Asymptotic Equisingularity and Topology of Complex Hypersurfaces, Int. Math. Res. Not., 18 (1998), 979-990. 
[Va] Varchenko, A. N., Theorems on topological equisingularity of families of algebraic varieties and families of polynomial maps, Izv. Akad. Nauk, 36 (1972), 957-1019.

[Ve] Verdier, J.-L., Stratifications de Whitney et théorème de Bertini-Sard, Invent. Math., 36 (1976), 295-312. 
